\title{
Epidemics and Literature (The Novel as A Model)
}

\author{
Ahmed G. Naef \\ Department of Arabic Language, Al-Maaref University College, Anbar, Iraq \\ dr.ah1975@uoa.edu.iq
}

\begin{abstract}
:
The literature in general and epidemiological literature especially represents the reflection of the health status of society and the health crises that the world has witnessed since ancient times. Those epidemics were the engine for many authors and novelists pens in particular. Thus, many novels touched on those diseases that caused violent shocks in the whole world, which left behind them many tragedies that remain stuck in the global memory. Since the novel is the most realistic literary art, it took the initiative to be the most prominent literary art that dealt with these topics and dealt with them, whether they were stories transmitted from reality or imaginary that predicted the future.
\end{abstract}

Keywords: Epidemics; Literature; Novel; Love; Tragedy

Crossref doi https://doi.org/10.51345/.v31i1.246.g141 


\title{
الأوبئة والأدب (الرواية انموذجا)
}

\author{
أ. أ.م.د. أحمد غالب نايف \\ قسم اللغة العربية،كلية المعارف الجامعة، الأنبار، العراق
}

drahmedghalib4@gmail.com, dr.ah1975@uoa.edu.iq

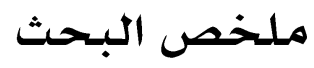

يمثل الأدب بشكله العام وخاصة أدب الأوبئة انعكاسا للحالة الصحية للمجتمع وما يمر بها العالم من أزمات صحية منذ القدم؛ حيث كانت تلك الأوبئة هي المحرك لأقلام الكثيرين من الأدباء والروائيين بشكل خاص، وتطرقت الكثير من الروايات إلى الحديث عن تلك الأمراض التي أحدثت هزات عنيفة في العالم بأسره، مخلفة وراءها الكثير من المآسي التي لا تزال عالقة برقعة الذاكرة العالمية. وبحا ان الفن الأدبي الأكثر واقعية هي الرواية فنجدها اخذت المبادرة بان تكون أبرز الفنون الأدبية التي تناولت هذه الموضوعات وعالجتها سواء كانت قصص منقولة من ارض الواقع ام متخلية استشرفت المستقبل.

الكلمات المفتاحية: الأوبئة، الأدب، الرواية، الحب، المأساة. Crossref doi https://doi.org/10.51345/.v31i1.246.g141 


\section{المقدمة}

الأدب ابن واقعه لا يغادر كبيرة منه ولا صغيرة حتى يسجلها وبها ان الأدب الروائي هو الأدب الأكثر واقعية فكانت الرواية اول الفنون تسجيلا للاوبئة التي تصيب المجتمع سواء اكانت صحية ام فكرية ام اقتصادية فتترصدها وتنقلها وتحذر منها وتطرح الحلول لها لا بل تسجل المواقف الإنسانية التي رافقتها ونحن اليوم بعد انتشار وباء كورونا او كوفيد 19 نبحث في الاععال الروائية التي عالجت اوبئة واقعية ام متخيلة استشرفت الزمن لنستشفي او هو نوع من المؤاساة لانفسنا. إنّ الأدب يصلح أيضًا لتوقيع نهاية الوباء وتحويل سو اد العالم إلى جرّد ذكرى سرعان ما تُنسَ، و النسيان ههنا أيضًا إنّما هو طريقة البشر في التغلّب على الكوارث التي تحلّ بهه.

\section{قراءة يِّ بعض الروايات التي تناولت الاوبئة}

في ازمة الجائحة (كوفيد 19 ) أو ما يسمى اختصار ا بـ(كورونا) التي ضربت العالم من مشرقه وحتى مغربه ومن شمالها المى جنوبه ولم تستن لا دولة متقدمة ولا متأخرة في الجوانب الصحية والثقافية ولا غنية ولا فقيرة وان اختلف تعامل المجتمع معها من عزل وحظر تجو ال تام وتوقف الحياة بنو احيها كافة لأشهر وما رافقه من جمود اقتصادي وانهيار في أسعار الطاقة و وبين من استخدم حظر القطيع معتمدا على ثقافة مجتمعاتهم والتزامهم بضو ابط الوقائية الصحية نجد بدا الانسان بالبحث عن ما يشغل وقت فراغه ويعده مهربا وعزاء وسلوى للنفس فضلا عن نوع من ثقافة الاطلاع. يعد الأدب ابن بيئته كيفما تكون يكون ينقلها في فنونه وانواعه لذلك انطلق منظرو الفن بنظريتهم الفن في خدمة المجتمع لذلك نرى أي تغييرات ومتغيرات وكوراث واويئة تصيب المجتمع بنواحيه كافة السياسية والاجتحاعية والثقافية والصحية تجد الأدباء يتناولوها سواء بشعرهم ام بنثرهم. وبها ان الفن الأدبي الأكثر واقعية هي الرواية فنجدها اخذت المبادرة بان تكون أبرز الفنون الأدبية التي تناولت هذه الموضوعات وعالجتها سو اء كانت قصص منقولة من ارض الواقع ام متخلية استشرفت المستقبل. 
لذا ارتبط الأدب العالمي بالاوبئة التي اجتاحت العالم وبتمعاته في القرون التاسع عشر والعشرين والواحد وعشرين مثل الطاعون والانفونزا الاسبانية والكوليرا والايدز في القرن العشرين و الإيبولا والكورونا في القرن الو احد و العشرين (1). سنتاول هنا بعض اهم الروايات التي جعلت الأوبئة كموضوع لها ودارت احداثها ضمن هذه الازمة الصحية وما كنت تحمل من دلالات الامل بالشفاء وانتهاء الازمات رغم صعوبة المواقف وتجلي واضح للعاطفة التي سيطرت على اغلب الرويات وموقف الحزن والشعور بالياس وهذا الصراع الثنائي بين الامل والياس ويدفع الرواية بجحالية عالية فتتقلفها الايادي فنراها تنتقل من المحلية الى العالمية فتترجم لاكثر من لغة وتعد من الروايات العالمية ولم يلتزم الباحث بالتسلسل الزمني للروايات لان لكل رواية تدور احداثها ضمن مرض وبائي يختلف عن الاخر.

\section{1 - 1 - 1واية الطاعون لالبير كامو:}

ولد الفيلسوف الوجودي والكاتب المسرحي والروائي الفرنسي الجنسية ألبير كامو في قرية الدرعان المعروفة ببلدة مندوفى بالجزائر ضمن مقاطعة قسنطينة في 7 نوفمبر-تشين الثاني 1913م - ونوفى في 4 يناير - كانون الثاني 1960م ، ولد يتيم الاب بعد مقتل والده -وهو في عامه الأول- اثناء الحرب العالمية الأولى، ومن ام اسبانية الأصل والجنسية معاقة تعاني من إعاقة الصم(2). حصل الروائي على منحة دراسية في قسم الفلسفة/ كلية الاداب لتفوقه ونبوغه مكنته من اكمال تعليمه بجامعة الجز ائر بعد ان انهاء دراسته الثانوية هناك (3). لقد شارك الفرنسيين نضالهم من الاحتلال الألماني فاصدر نشرة باسم الكفاح تحمل اسم الخلية التي ينتمي اليها والتي تحولت فيما بعد المى صحيفة (الكفاح) اليومية بعد تحرير باريس واصبحت الناطق الرسمي للمقاومة الشعبية. عكست اعماله المسرحية و الروائية ما امن به من الفلسفة الوجودية والموت والحب والحرية والمقاومة والثورة، ويذلك يعد البير كامو ثاني اصغر اديب نال جائزة نوبل للآداب(4). 
تعد رواية (الطاعون) التي تدور احداثها حول انتشار الوباء في مدينة جزائرية الا وهي مدينة وهران عام 1940 هذه المدينة الشهير بجمالها وروعة طقسها وطبيعتها الخلابة وتمازج ثقافاتها واطلالتها الجميلة على البحر الأبيض المتوسط مما أهلها لتكون ميناء ميزا فجعلها مدينة اقتصادية هامة والتي كانت محطة لمختلف الثقافات العربية والتركية والاسبانية والفرنسية بالتعاقب حسب الجيوش الغازية وقوتها لذلك تجد فيها الثقافة المشرقية امتزجت بالثقافة الاوربية. اتخذ الاديب ألبير من الاحياء الاوربية في هذه المدينة مكانا لإحداث روايته لتحكي لنا كيف قام المسؤولون عن المدينة بإغلاقها لأشهر عدة للحد من إصابات وتفشي وباء يودي بحياة الكثير من سكانها(5). وتحدث الرواية عن نكر ان المسؤولين المحليين في اول الامر وعدم اعتر افهم بالأرهاصات الأولى للمرض وهي انتشار جثث الفئران النافقة والمصابة بالطاعون في الطرقات، ويعكس لنا الراوي، الدكتور برنار ريو، البطولة الكبيرة التي يقوم بها الجيش الابيض من الفرق الطبية، تلك البطولة الصامتة التي لا يعرفها الا من اقترب منهم وعمل معهم (6)، لقد رصد كامو تلاحمهم وتآزرهم رغم انتحائهم إلى طبقات اجتحاعيّة خختلفة. كا تناولت الرواية الكثير من الاحداث عن طريق السارد في الرواية الطبيب برنار ريو، والغريب جان تارو والتي كانت محورا في الاحداث والتي تعكس رؤية الكاتب لا يسعفنا حجم البحث لسرد الكثير من

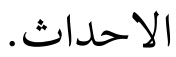

وفي ختام الرواية فانها تحمل رسالة مفادها، بـ"أننا نعلم الآن أنه إذا كان هناك شيء يمكن أن يتوق له المرء دائما، ويناله أحيانا، فهو الحب الإنساني (7)".

\section{2-روايـة عيون الظلام للروائي الأمريكي دين كونتز}

رواية عيون الظلام للروائي الأمريكي لدين كونتز الصادرة عام 1981م وهي رواية باللغة الإنكليزية ترجمت حديثا للعربية - خيالية(8) اثارت كلاما كثيرا حولها خاصة بعد ان ظهر وباء كورنا المستجد والتي حملت في طياتها إشارة لفايروس يقضي على البشرية كسلاح بايلوجي يخرج من مدينة ووهان الصينية التي 
يحمل اسمها ووهان 400 وان كانت في طبعاتها الأولى ان الفايروس الذي ينتشر في العالم من ينطلق من مدينة كوركي الروسية لكنها غيرت في الطبعة الأخيرة(9) إلى ووهان الصينية. واذا عقدنا موزانة بين الفايروسين كورونا الواقعي ووهان 400 الخيالي او الافتراضي في الرواية رغم التقارب الحدثي والمكاني في انطلاق هذا الفايروس من مركز الأبحاث الوطنية الصينية في ووهان وسرعة انتشاره وقضاءه على البشرية بنسبة عالية الا انه يختلف عن كورنا الذي كما يزعم العلماء بانه انتقل من الحيوانات الى البشر بعد ان طوره نفسه، فضلا عن ان كون كورنا لم يقض الا على 2 ٪ من المصابين في العالم كله في حين ان الفايروس الافتراضي يقضي على البشرية بها يقارب 99 ٪٪ وانه يصيب خلايا المخ اذ ينتقل الفيروس إلى جذع الدماغ، وهناك يبدأ في إفراز مواد سامة تتلف الدماغ فانه يدمر مركز الدماغ المسؤولة عن وظائف الجسم كما يختلفان أيضا بسرعة الإصابة اذ نجد ان كورونا الحقيقي تترواح مدة الإصابة وحتى الشفاء ما بين 14-28 يوم في حين ووهان 400 الافتراضي لا تتجاوز مدة الإصابة فيه ووفاة المصاب الا اثنتا عشرة ساعة فقط. تتحدث الرواية عن ام تبحث عن ابنها المتوفى وسبب وفاته لتكتشف ان ابنها لا يزال حيا لكنه قد حقن بفايروس ووهان 400 من قبل الجيش الأمريكي وتحويله الى سلاح فتاك يقضي على الأعداء بسرعة عالية ومثالية. تعد هذا الرواية الان في العالم في المرتبة الأولى من حيث عدد القراء ـ وجاء ازدياد الطلب عليها بعد انتشار وباء كورونا المستجد او (كوفيد 19)(10) واذا عدنا المى سبب اختلاف اسم الفايروس والمدينة التي اكتشف فيها وتحو لها من مدينة كوركي 400 أو (جوركي) الروسية الى مدينة ووهان الصينية وحمله لاسمها ووهان 400 ريحا يعود ذلك لأسباب سياسية وهذا ما يراه جميع من تطرق لموضوع هذه الرواية في مقالاتهم التي تناولت الموازنة بين الفايروسين.

\section{3- رواية الحب يْ زمن الكوليرا د غابريسيل غارسيا ماركيز}

غابرييل غارسيا ماركيز، روائي وصحفي وناشر وناشط سياسي كولومبي ولد في أراكاتاكا، ماجدالينا في كولومبيا سنة 1927. تحصل على نوبل للآداب سنة 1982 ـ من أهم أعحاله "مئة عام من العزلة" وله أيضا 
"ذكريات غانيات حزينات" و"خريف البطريرك" روايته موضوع الدراسة التي صدرت عام 1985 تدور حول حب بدأ في سن المراهقة لينتهي بالزواج بعد بلوغهم العقد السابع من أعلارهم ففي نهاية قرن التاسع عشر الماضي وبداية القرن العشرين، يهاول الروائي ان يضعنا في زمن الحروب والأوبئة، في زمن يصحو فيه مجتمعه من بعد سبات القرون الوسطى ليشاهد إنجازات العلم وتقدمه في القرن العشرين وانعكاسات هذا الإنجازات على الصعيد الاجتماعي والإنساني. وماركيز يعالج في روايته الحب المحاصر بالحروب الاهلية والاويئة وقد استوحى فكرة روايته من قصة حب بين أمه وأبيه ثم أضاف لها حبكة بقصة قرأها في الصحف عن حب كان بين عجوزين وانتهى نهاية مأساوية

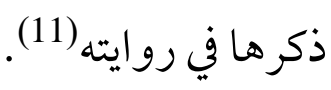
رواية الحب في زمن الكوليرا غنية بالسرد وفنونه التي جعلت منها متميزة في كل شيء، تتحدث عن شاب في الثامنة عشرة من عمره اسمه فلورينتيو تعرف على فتاة تدعى فيرمينا بمحض الصدفة، فلورينتيو الموظف في دائرة البريد كان محط اعجاب الفتيات، فهو الأكثر شهرة في الوسط الاجتماعي لذلك عاش مغامرات كثيرة مع فتيات المدينة ليعتزل كل ذلك بعد تعرفه فيرمينا(12). ربيا صدفة جمعته بها عندما أوصل برقية الم شخص بلا عنوان اسمه لورينثو داثا والد فيرمينا، جمعته نظرة ساحرة لصبية وهو يمر عبر النافذة عندما كانت تجالس امراة مسنة تقر آن في كتاب، هذه النظرة العابرة اشلت تفكيره فلورنتينو وجعلت قلبه ينسى كل الفتيات ويتعلق بها ويولد داخله حب لم ينته بعد مرور خمسة عقود من الزمن تقريبا(13) من كانت حياة العاشقين خالية من المفاجات تمثل حبا مثاليا مستقر الأعوام، كان خلالها يتبادلان الرسائل الغرامية ووعود الوفاء والبقاء على عهود الزواج، الى ان علم الاب بعلاقة ابنته فتحولت حياة العاشقين الى جحيم وخاصة بعد قرار الاب المغادرة من المدينة الم الابد هبا من العار الذي الحقته به ابتته المتمردة، ومحاولة إيجاد زوج مناسب لما، وهنا يحاول ان يعكس لنا ماركيز في سرد صرامة الجل اللاتيني في الحفاظ على عرضه (14). 
ان سرعة الاحداث في الرواية وزواج فيرمينا من الطبيب الارستقراطي خوفينال اوربنيو صاحب الشهرة الواسعة بالمدينة والمنطقة ، ضغط الاحداث والظروف الاجتحاعية للفتاة وقدسية الزواج الكاثوليكي وانجاب الأبناء جعلتها تنسى موظف البريد ذلك المسكين العاشق في مقابل ذلك نجد فلورينتينو يثابر بكل جهد ليصنع لنفسه اسما لامعا ويكوّن ثروة ضخمة تجعله قادرا على استعادة حبيبته من زوجها(15). في سرد واقعي سحري تتشابك الاحداث بطريقة مشوقة ، يتوفى الطبيب خوفينال اروبينو بعد نصف قرن من الزواج بسبب محاولته الفاشلة للامساك ببيغائه الوقحة، فيذهب فلورينتينو الى الجنازة ليقدم بين يدي حبيته الأبدية عهد الوفاء اذ قال لها: "لقد انتظرت هذه الفرصة لأكثر من نصف قرن لأكرر لك مرة أخرى قسم وفائي الابدي وحبي الدائم"(16). اثبت ماركيز في روايته ان الحب الحقيقي ينقل الانسان من الظلام الى النور ومن اليأس الى الامل ومن الحزن الى السعادة ومن الضعف الى القوة ومن الفقر المى الغنى، ومن انسان بسيط الفهم الى انسان مفكر ومبدع، وان ما يمر به من ظروف يبقى امل الحب ينير طريقه ويمده باسباب الحياة. نجح ماركيز في سرده الفريد، ذاك السرد الذي يجمع الواقعية والسحر (17)، الغريب بالعجيب الذي استند على فلسفة العلاقة الغرامية المرتكزة على الأبدية كأساس ها، هذه العلاقة تقوم على الثنائيات الضحك و البكاء، الخوف والأمان ،الحزن و الفرح، الحضور والغياب، الانتصار والهزيمة ليثبت للمتلقي العام -عبر الازمانبطريقة جميلة ولطيفة وبطريقة واضحة لا غبار عليها ان الحب مستمر في كل الأزمنة، وفي كل الظروف، وفي مراحل العمر كلها، فلا المراهقة ولا سطوة الاب ولا الشيخوخة تمنع الحب الصادق فهو شعور جميل ساحر وسحي بنفس الوقت لا يمكنك الا الضياع و التحاهي فيه. يقول النّاقد المكسيكي إيحانويل كاربايو في تعليقه على الرواية إنه عندما قر أها في نسختها الأولى وجد نفسه أمام واحدة من أعظم الرّوايات في القرن العشرين. ويضيف النّاقد "أن ماركيز بأعماله الرّوائية قد أقام إلى جانب روائين آخرين أُسس وقواعد الواقعية السحرية في أميركا اللاتينية من خلال تحليله العميق لو اقع الإنسان الأميركيّ اللّاتيني ومعالجة الأساطير وتوظيفها بطريقة ذكية"(18). 
"الحب في زمن الكوليرا" رواية السحر تبطنها الحية والجنون عي واقعية لدرجة ان يتحول الحيال فيها وكأنه واقعا يتجسد امامنا نلمسه ونتلمسه بايدينا، نجد انفسنا غرقى فيه يخطف عقولنا وقلوبنا من البداية وحتى

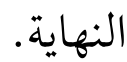
في هذه الرواية الجمميلة كل شيئ مكن الحدوث حتى المستحيل مككن الحدوث فهو يغدو مككنا وكأنك تقول ان هذه القصة قصة حب حقيقية جمعت شخصين كتب عليها الفراق لكنها احبا بعضها بطريقة ما، عاشا بعيدين لكنها اجتمعا متاخرين ـ الرواية قامت على دائين مختلفين فداء الكوليرا يفرق المحبين وداء الحب يمعهم هذه هي رواية الحب في زمن الكوليرا.

\section{الخاتمة والنتائج}

في صراع الحياة اليومي وما يتبعئ به العلماء والمفكرون من انهيار للحضارة الحالية ومنذ ثمانينيات القرن المنصرم والحروب النووية وثقب الامزون والأزمات الاقتصادية والثورات الشعبية والحروب العسكرية كلها كانت تتحدث عن نهاية العالم وكما صورتها السينما الهوليودية، لكن العالم لم ينهار حتى بدات احساديث علمية

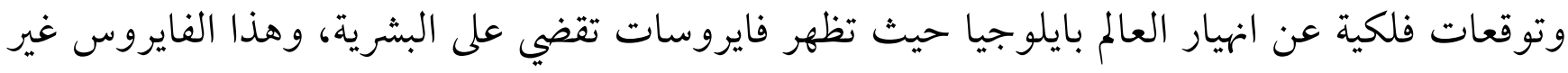
مرئي يداهم البيوت والمدائن ولا يخلف سوى جثث الموتى والرعب حتى يتحول هذا الرعب الى قاسم مشترك يك يكي

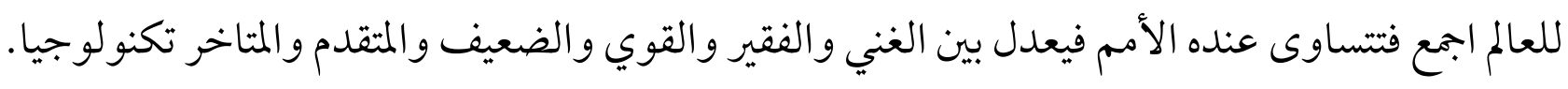
لم تكن هذه الفايروسات من احاديث العلماء فقط وانما ظهرت أيضا على السنة الأدباء في ادابهم والرواية

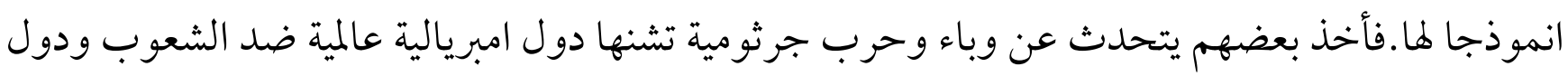

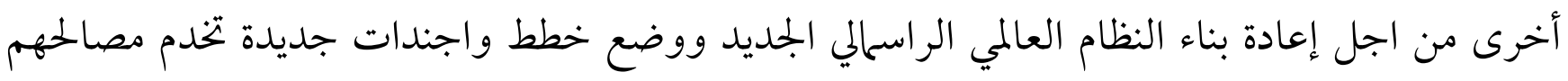
الاقتصادية، فكانت الرواية كفن مسكن لكثير من هذه الاوجاع وصوت لأؤلك الموتى الذين اصمت

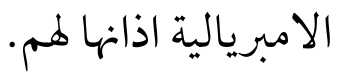


الهدف من ظهور ادب الأوبئة لم يكن مقصورا على تتبع وتحليل أسباب ظهور وانتشارها ووان كان فهو ليس الهدف الرئيس من اهداف الأدب.

فالأدب عموما و الرواية والقصة خاصة هما وسيلة للتذكير بان العقل الإنساني ما زال ولا يزال موجودا، فالأدب عملية اصلاح وتوعية وارشاد لمو اجهة الامر اض والاوبئة. ان قابل الأيام وقادمها يممل الكثير من الروايات والقصص التي تتحدث بواقعية كبيرة قصص ابطالها حقيقيون عاشوا وتعايشوا مع هذه الجائحة (كوفيد 19 ) وما رافقها وصاحبها من موافق إنسانية مؤلمة واليمة ومواقف أخرى وصف بالبطولية والشجاعة للجيوش البيضاء التي كانت خط الدفاع والصد الأول التي تصدت بارو احها لتنقذ البشرية جمعاء.

\section{الهوامش}

(1) سياسية الأدب: جاك رنسيير، ترجمة د. رضوان ظاظا، بيروت، مركز دراسات الوحدة العربية، 2010م، ص.15.

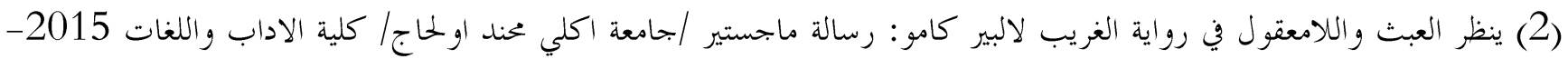
تصنه

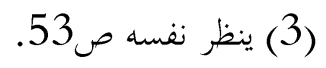

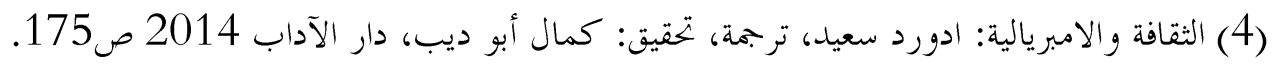

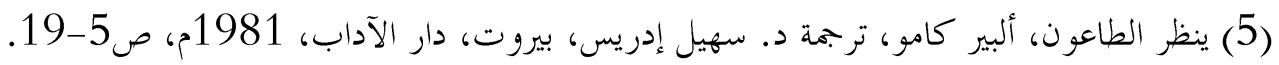

$$
\begin{aligned}
& \text { (6) ينظر نفسه ص 35-44. } \\
& \text { (7) ينظر نفسه ص301. }
\end{aligned}
$$

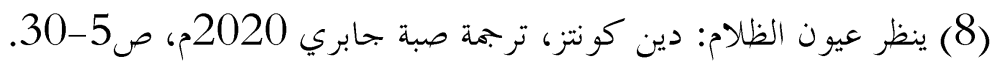

$$
\text { (9) ينظر عيون الظلام: ص 265-269. }
$$

وينظر: (2008) Afterword for The Eyes of Darkness Koontz, Dean صفحات: 374-369 صنات

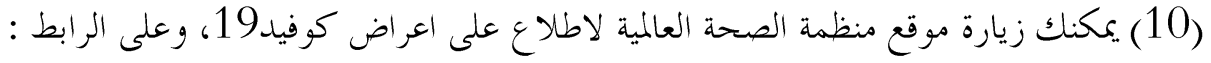

https://www.who.int/ar/emergencies/diseases/novel-coronavirus-2019/advice-for-public/q-a-coronaviruses

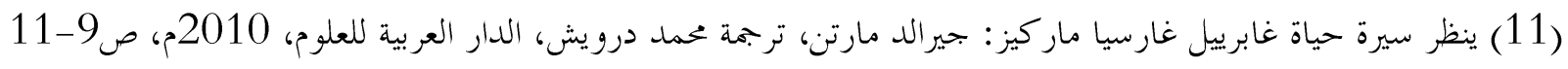

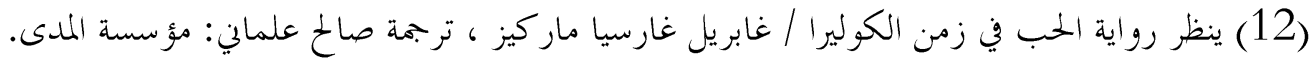




$$
\begin{aligned}
& \text { (13) ينظر نفسه ص34. (13) - (13) }
\end{aligned}
$$

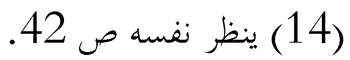

$$
\begin{aligned}
& \text { (15) - (15) ينظر نفسه ص نفس ص } 98 .
\end{aligned}
$$

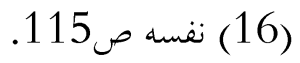

$$
\begin{aligned}
& \text { (17) ينظر في الواقعية السحرية : الدكتور حامد أبو احمد، ص 261-271. } \\
& \text { (18) نفسه المقدمة ص4. }
\end{aligned}
$$

